\title{
Diagnostic Performance of MRI Diffusion Weighted Heterogeneity Index to Discriminate Brain Metastases from Normal Brain Tissue in Hybrid $\mathrm{PET} / \mathrm{MR}$
}

\section{Bahattin Özkul ( $\sim$ bahozkul@yahoo.com )}

Atlas University: Istanbul Atlas Universitesi https://orcid.org/0000-0003-3339-8329

\section{Bedriye Koyuncu Sokmen}

Demiroglu Bilim University: TC Demiroglu Bilim Universitesi

Ibrahim Halil Sever

Demiroglu Bilim University: TC Demiroglu Bilim Universitesi

Nagihan Inan Gurcan

Demiroglu Bilim University: TC Demiroglu Bilim Universitesi

\section{Research Article}

Keywords: ADCCV, Brain, Heterogeneity index, Metastasis, PET/MRI

Posted Date: June 11th, 2021

DOl: https://doi.org/10.21203/rs.3.rs-595957/v1

License: (c) (1) This work is licensed under a Creative Commons Attribution 4.0 International License.

Read Full License 


\section{Abstract}

\section{Introduction}

To evaluate diagnostic performance of apparent diffusion coefficient (ADC) heterogeneity index to differentiate brain metastasis (BM) from normal appearing brain parenchyma (NABP) and to find out the correlation between 2-[18 F]-fluoro-2-deoxy-D-glucose $\left({ }^{18} \mathrm{~F}-\mathrm{FDG}\right)$ standardized uptake value (SUV) and ADC heterogeneity index derived from hybrid PET/MRI.

\section{Methods}

Whole-body PET/MRI was performed to evaluate proven 40 BM of 18 oncology patients (9 females, 9 males; mean age $61 \pm 16$ years), sourced from different primary cancer. Brain sequences, which were dixon and diffusion weighted imaging (DWI) protocols with simultaneous PET were used to calculate coefficient of variance of the $A D C\left(A D C_{C V}\right)$ and SUVmax. All images were assessed by three radiologists and the same size of VOI was placed on BM and NABP. Inter-rater reliability was tested by inter-class correlation (ICC). The correlation of $A D C_{C V}$ and SUVmax and the differences in ADC values and SUVmax between $B M$ and NABP were investigated.

\section{Results}

The excellent consistency was found between raters at $A D C$ mean (0.972) and $A D C_{C V}$ (0.995). There was a strong correlation between $\mathrm{ADC}_{\mathrm{CV}}$ and SUVmax $(r=0.763)$ and a slight inverse correlation between $A D C m e a n$ and SUVmax $(r=-0.122)$. A statistically significant difference between BM and NABP was determined for $A D C_{C V}(p<0.001)$ and SUVmax $(p<0.001)$. An area under the curve (AUC) of 0.960, 0.998 and 0.574 were obtained with ROC analysis of SUVmax, $A D C_{C V}$ and $A D C$ mean, respectively.

\section{Conclusion}

$\mathrm{ADC}_{\mathrm{CV}}$ may be considered as a potential biomarker that quantitatively discriminates $\mathrm{BM}$ from NABP with excellent interrater reliability.

\section{Introduction}

Brain metastasis (BM) is associated with poor survival outcomes and poses distinct clinical challenges. Lung cancer, renal cell carcinoma, breast cancer, melanoma and colorectal cancers are the most common causes of BM [1]. Due to great variation in imaging appearances, these metastases present a common diagnostic challenge which can affect patient management.

Computed tomography (CT) and magnetic resonance imaging (MRI) are the key imaging modalities used in the diagnosis of BM. In some cases, advanced imaging techniques including proton magnetic resonance spectroscopy (MRS), contrast enhanced magnetic resonance perfusion (MRP), diffusion 
weighted imaging (DWI), and diffusion tensor imaging (DTI) may help for the diagnosis [2]. Although these imaging techniques are essential in the diagnosis, using quantitative data may lead to improved detection of BM.

DWI is a fast, non-contrast MR technique that indicates the random microscopic motion of free water molecules. It is widely appreciated as a qualitative tool in the examination of the central nervous system (CNS). Apparent diffusion coefficient (ADC) is a measure, calculated using DWI and reflects the magnitude of diffusion quantitatively.

Tumors are heterogeneous because of the spatial variation in the cellularity, angiogenesis, extracellular matrix and necrosis $[3,4]$. Higher intratumoral heterogeneity is related with poor prognosis due to its aggressive behavior [5-7]. Thus, measuring of tissue heterogeneity may be helpful in the detection of tumors and selection poor prognostic patients for more intensive therapy. There are various methods using complex textural analysis in the detection of tissue heterogeneity [8]. Of all these, the coefficient of variation $(C V)$ is easily calculated and shows relative variability. In line with this, $A D C_{C V}$ as a reliable heterogeneity index was used in different studies [9-12].

Positron Emission Tomography (PET)/MRI is a new imaging technology that allows for PET and MRI scans to be acquired simultaneously. Although MRI is the standard neuroimaging technique for detection of tumors and the surrounding anatomical structures in the brain, PET aids to complement MRI in lesion grading, tumor extent delineation and evaluation of the treatment response. Allowing both structural and functional evaluation of tumors in one single scan makes PET/MRI more popular in oncology imaging.

The primary target of our study was to determine the diagnostic performance of $A D C_{C V}$, as a heterogeneity index, to differentiate BM from normal appearing brain parenchyma (NABP), as compared to conventional MRI metrics used in daily routine. A secondary target of this research was to investigate correlation of $\mathrm{ADC}_{\mathrm{CV}}$ and standard uptake value (SUVmax) measured on PET-MRI. To the best of our knowledge, this is the first study that evaluates the diagnostic performance of $\mathrm{ADC}_{\mathrm{CV}}$ in brain metastases and its correlation with SUVmax on PET/MRI hybrid system.

\section{Material And Methods}

\section{Study Design}

347 consecutive adult patients with known malignancies who underwent PET/MRI between January 2017 and September 2019 were evaluated. Forty-five patients who had BM were enrolled in this retrospective single center study. The patients who has multiple lesions (if there is no enough NABP), a massive brain edema, a history of radiotherapy and no 2-[ $\left.{ }^{18} \mathrm{~F}\right]$-fluoro-2-deoxy-o-glucose $\left({ }^{18} \mathrm{~F}-\mathrm{FDG}\right)$ uptake were excluded from the study. Decision of BM was given if lesions growth at least two imaging methods in the follow-up imaging (3-6 months) or proven with biopsy (single lesion). Thus, 40 lesions of 18 
patients were included and analyzed for this study (Fig. 1). All primary malignancies were proven histopathological by biopsy or surgery.

\section{Image acquisition}

Patients fasted at least 6 hours before starting examination and injection of ${ }^{18} \mathrm{~F}-\mathrm{FDG}$ was given if blood glucose levels were $<140 \mathrm{mg} / \mathrm{dL}(7.77 \mathrm{mmol} / \mathrm{L})$. All scans were performed with the patient in the supine position on the 3 Tesla Biograph mMR scanner (Siemens Healthcare, Erlangen, Germany) using a 16channel head and neck surface coil and three 12-channel body coils and the total scanning time was $60 \pm 3$ minutes. The whole-body images, which cover the entire body from head to heel, were obtained in five to six bed positions according to body-mass index (BMI) of patient. PET attenuation correction was performed using four compartment model attenuation map calculated from a Dixon-based volumetric interpolated breath-hold examination (VIBE) sequence. The MRI protocol included sequences as below: T1-weighted slice-selective Turbo Flash (TR/TE, $1600 \mathrm{msec} / 2.5 \mathrm{msec}$ ) in the axial plane, free breath diffusion-weighted imaging using the echo planar imaging technique (EPI) (TR/TE, $12000 \mathrm{msec} / 78$ $\mathrm{msec}, \mathrm{b}=0 \mathrm{~s} / \mathrm{mm}^{2}$ and $800 \mathrm{~s} / \mathrm{mm}^{2}$ ) in the axial plane and T2-weighted single-shot echo train (HASTE) (TR/TE, $1500 \mathrm{msec} / 87 \mathrm{msec}$ ) in the coronal plane. Contrast enhanced protocol including the breath-hold 3D VIBE sequence (TR/TE, $4.56 \mathrm{msec} / 2.03 \mathrm{msec}$ ) in the arterial, portal venous and equilibrium phases covering whole-body in the axial plane was performed with using a weight-adapted gadolinium-based contrast agent and all sections were then combined.

\section{Image analysis}

All image datasets were transferred to the dedicated Syngo.via PET/MRI workstation (Siemens Healthcare) and images were assessed separately by three radiologists (I.H.S, B.K.S and N.I.G) with at least 6 years of experience who were blinded to the patients' information. A volume of interest (VOI) was placed manually on axial PET images and all three planes were controlled for ensuring to not overflow the limits of the lesions. The VOI was coregistered and placed on ADC images overlapping with PET images. Manual correction was used to fine tune when the images were not overlapped. For each determined lesion, a similar size of VOI was used on NABP (Fig. 2). Care was taken to keep away from edema, blood vessels and cerebrospinal fluid and for preventing bias, white matter, which did not include sulcus, was used to evaluate NABP. SUVmax (SUV of the hottest voxel within a defined VOI), which is easy to use and operator independent, was calculated automatically and measured on PET images. The mean (ADCmean) and standard deviation (SD) of $A D C\left(A D C_{S D}\right)$ were calculated automatically by software for each measurement. $A D C_{C V}$ was created by dividing the $S D$ by the $A D C$ mean.

\section{Statistical analysis}


IBM Statistical Package for the Social Sciences (SPSS ver. 21 for windows, Chicago, IL, USA) software was used for all statistical analysis. Intra-class correlation coefficient (ICC) was used for determining inter-rater reliability in $A D C$ mean, $A D C_{S D}$ and $A D C_{C V}$. The ICC value ${ }^{2} 0.50,0.51-0.75,0.76-0.90$ and $>0.90$ were evaluated as indicating poor, moderate, good and excellent reliability, respectively. The fitness of numeric data set to normal distribution was determined by the Shapiro-Wilk test. Due to non-normal distribution, correlation between SUVmax and $A D C_{C V}$ was tested by Spearman's rank correlation. Wilcoxon signed rank test was carried out to measure differences between BM and NABP for all variables. Receiver operating characteristics (ROC) analysis based on histopathological results was performed to determine cut-off value, which differentiate BM from NABP, by the Youden index. The area under the curve (AUC), sensitivity and specificity were calculated for each variable. A p-value 0.05 was accepted as statistically significant.

\section{Results}

\section{Patients demographic}

Eighteen patients ( 9 female, 9 male) with proven BM were included in the study. The patients aged between $28-87$ (mean \pm standard deviation; $61.4 \pm 16.6$ ) years. 14 patients $(78 \%$ ) also had metastases in other locations besides the brain. $40 \mathrm{BM}$ were analyzed with VOI mean $3,57 \pm 4,13 \mathrm{~cm}^{3}$. As identified in table 1, breast (invasive ductal carcinoma, 4 patients; invasive lobular carcinoma, 4 patients), lung (adenocarcinoma, 5 patients; small cell carcinoma, 2 patients), kidney (renal cell carcinoma, 2 patients) and skin (malignant melanoma, one patient) were the primary source of tumors for BM.

\section{Interrater reliability}

There was an excellent consistency between raters at $A D C$ mean, $A D C_{S D}, A D C_{C V}$ and SUVmax with ICC 0.972 [95\% confidence interval (Cl] 0.952-0.984), 0.990 (95\% Cl 0.983-0.994), 0.995 (95\% Cl 0.992-0.997) and 0.993 (95\% Cl 0.989-0.996), respectively.

\section{Correlation with SUVmax}

For all values, the mean of three raters was calculated and presented as $A D C m e a n, A D C_{C V}$ and SUVmax. According to Spearman's correlation coefficient, there was a strong correlation $(r=0.763, p<0.000)$ between $A D C_{C V}$ and SUVmax when all measurements included (BM+NABP). A slight inverse correlation was found between ADCmean and SUVmax ( $r=-0.122)$.

\section{Differences between BM and NABP}


A statistically significant difference between $B M$ and NABP with $p<0.001$ value was found for $A D C_{C V}$ and SUVmax. There was no statistically significant difference for $A D C$ mean $(p=0.253)$. The mean $\pm S D$ values of $A D C m e a n, A D C_{C V}$ and SUVmax of all lesions were presented in the table (table 2).

An AUC for SUVmax of $0.96(95 \% \mathrm{Cl} 0.91-1.00, \mathrm{p}<0.001)$ was yielded with ROC curve analysis. $\mathrm{ADC}_{\mathrm{CV}}$ (AUC:0.998, $\mathrm{p}<0.001$ ) had higher AUC with a smaller standard error and a narrower confidence interval than ADCmean (AUC: $0.574, \mathrm{p}=0.256)$ and SUVmax. The sensitivity and specificity of $\mathrm{ADC}_{\mathrm{CV}}(97.5 \%$, $97.5 \%$, respectively) were higher than SUVmax $(95 \%, 95 \%)$ and ADCmean $(55 \%, 70 \%)$. The cut-off value based on the maximum Youden index to determine differentiation between BM and NABP was ${ }^{3} 4.00$ for SUVmax, ${ }^{2} 0.8410^{-3} \mathrm{~mm}^{2} / \mathrm{s}$ for $A D C$ mean and ${ }^{3} 0.10$ for $A D C_{C V}$. Cut-off value, sensitivity, specificity, confidence interval and standard error of all values were summarized in the table (table 3 ).

\section{Discussion}

In this study, we investigated the role of $A D C_{C V}$ derived from $P E T / M R I$ as a heterogeneity index in discriminating $B M$ from NABP. The main finding of this study was that, $A D C_{C V}$ is more effective to discriminate $B M$ from NABP compared to conventional $A D C$ parameters. Besides, $A D C_{C V}$ was highly correlated with SUVmax from simultaneous derived PET/MRI system.

Integrated PET / MRI scanners with the recent developments, new opportunities have emerged for quantitative molecular imaging. PET / MRI provides multimodal analysis of concurrently acquired functional parameters that can contribute to better characterization of tumor biology and also help identify markers to predict response to therapy $[13,14]$.

Due to the high ${ }^{18} \mathrm{~F}-\mathrm{FDG}$ uptake of the cerebral cortex and the low spatial resolution of PET imaging, FDG $\mathrm{PET} / \mathrm{CT}$ imaging has limitations, especially in the detection of small metastases in the brain. Sensitivity of FDG PET/CT in brain imaging is low. In retrospective comparative studies, it is stated that FDG PET/CT imaging at the time of diagnosis can capture up to $61 \%$ of metastatic brain lesions that can be detected by MRI [15]. Therefore, PET / MR imaging may be preferred in brain metastasis scanning because of the high soft tissue contrast of MR imaging.

SUVmax measured by PET and ADC measured by MRI allow assessment of water diffusion and glucose metabolism in tumor cells. The results of the present study show that $A D C_{C V}$ exhibits an improved correlation with SUVmax. Moreover, it provides better quantitative separation between BM and NABP, as compared to common MRI metrics.

In this study, $A D C m e a n$ showed a significant negative correlation with SUVmax; however, $A_{C D} C_{C V}$ showed higher correlation with SUVmax than ADCmean parameter. There are previously reported oncologic studies of the inverse correlation found between ADC and SUV. Several of these studies reported significant strong inverse correlation between ADCmean and SUVpeak in rectal cancer [16], a significant 
inverse correlation between ADCmean and SUVmean in gastrointestinal stromal tumor [17], and recently an inverse correlation between ADC and PET SUV in liver tumors [18].

Tumor heterogeneity consists of marked differences in cell mix, size, and arrangement. Heterogeneity also plays a role in micro-environmental factors (including oxygenation, $\mathrm{pH}$, interstitial pressure, blood flow), metabolism, and gene expression. This deep heterogeneity is extremely important for prognosis, treatment planning, and drug distribution, which ultimately affects patient outcomes. There are a number of ways to investigate tumor heterogeneity, some of which include functional and molecular imaging, which can be applied to clinical data [19].

The characterization of tissues can be improved using histogram-based assessments of the distribution of $A D C$ values. Histogram approaches have multiple advantages, including volume-of-interest (VOI) assessments, thus avoiding the subjectivity that is inherent with ROI placements. Importantly, histograms can provide additional metrics that reflect the texture of lesions, thereby allowing heterogeneity of ADC distribution within tissue to be assessed [20]. Tissue heterogeneity analysis is rapidly evolving by various methods. Despite most of the tools currently offered are often complex and computationally costly, it is an easy to calculate texture parameter of $A D C_{C V}$. Several studies have used $C V$ as an index of heterogeneity in recent years.

In a study in liver metastasis, the results of this study show that $A D C_{C V}$ can significantly distinguish between liver metastasis and normal-appearing liver [9]. Similar to our study, there was a good correlation between $A D C_{C V}$ and SUV peak in this study. Significant differences in CV diffusion index was found in another study about hepatocellular carcinoma in fresh liver explants [21].

PET / CT and DWI share applications in clinical oncology. While both SUV and ADC correlate with cellularity, SUV is also associated with several other pathological markers such as mitotic count, presence or absence of necrosis [22]. For this reason, PET / MRI oncological evaluation is also valuable when these two parameters (SUV and ADC) are obtained together in the same examination. In a study by Nakajo et al. [23], 44 patients with breast cancer underwent preoperative PET/CT and DWI within an average of 17 days between examinations, and both SUVmax and ADC were significantly associated ( $p<$ 0.05 ) with histologic grade (independently), nodal status, and vascular invasion. This finding suggests that SUVmax and ADC correlate with several of the pathologic prognostic factors and that both values may have the same potential for being predictive of the prognosis of breast cancer.

In oncology, imaging has a very important place in evaluating response to treatment. For this reason, many studies are aimed at understanding the structure and heterogeneity of the tumor. Therefore, it is essential to develop quantitative imaging methods and objective biomarkers to improve the diagnosis of brain metastasis. As a volume-independent index of heterogeneity, $A D C_{C V}$ can be considered as a potential biomarker that quantitatively differentiates BM from NABP. Tissue heterogeneity has been proposed as a basis for a biomarker for tumors $[3,4,24]$. 
This hybrid PET / MRI study shows a significant negative correlation between metabolic activity on ${ }^{18} \mathrm{~F}$ FDG PET and water diffusion over DWI in brain metastasis, possibly because both parameters are directly related to tumor cellularity. The correlation found between SUVS and $A D C$ mean, $A D C_{C V}$ values supports the idea that high cellularity due to tumor proliferation results in greater metabolic activity and restricts water diffusion.

This study has several limitations. First, this was a retrospective study and performed on a relatively small study population. Another limitation was the difficulty in determining the limits of the lesions due to the limited resolution of PET. The accuracy of the results obtained from our study should be supported by using different software in larger patient groups and with multi-center studies. The last limitation of our study was that brain metastases originated from different sources.

\section{Conclusions}

In conclusion, using PET/MRI instead of PET/CT decreases radiation dose, however radiation exposure caused by short term follow-up imaging of oncology patients continue to be an issue. Although determining brain metastases compared to normal brain parenchyma are not the main challenge in oncologic patients, $A D C_{C V}$ may be helpful to clinicians for avoiding further radiation exposure of patients and for managing patients when using contrast media is contraindicated. Moreover, it would be easy to implement $A D C_{C V}$ in a clinical setting. Future studies that will blindly and independently identify $B M$ in NABP using PET ${ }^{18}$ F-FDG SUV and DWI $A D C_{C V}$ will present potential to investigate $A D C_{C V}$ as a biomarker for BM.

\section{Declarations}

\section{Funding:}

This research did not receive any specific grant from funding agencies in the public, commercial, or notfor-profit sectors.

\section{Conflict of interest:}

The authors have no relevant financial or non-financial interests to disclose.

\section{Ethics approval:}

This study was performed in line with the principles of the Declaration of Helsinki. Approval was granted by the Ethics Committee of Demiroğlu Bilim University (Date. 27.10.2020/No. 2020-20-04). 


\section{Consent to participate:}

Informed consent was obtained from all individual participants included in the study.

\section{Consent for publication:}

Patients signed informed consent regarding publishing their data.

\section{Availability of data and material:}

The datasets analyzed in the current study are available from the corresponding author on reasonable request.

\section{Code availability:}

Syngo.via (Material no:10496180, Serial no:130408)

IBM SPSS V.21 (Authorization code: 4b37d8fed81bc545db11)

\section{Author's contribution:}

Conceptualization: [Bahattin Özkul]; Methodology: [Bahattin Özkul], [Bedriye Koyuncu Sökmen]; Formal analysis and investigation: [Bahattin Özkul], [ibrahim Halil Sever], [Bedriye Koyuncu Sökmen]; Writing original draft preparation: [Bahattin Özkul], [Bedriye Koyuncu Sökmen], [ibrahim Halil Sever]; Writing review and editing: [Bahattin Özkul], [Nagihan İnan Gürcan]; Supervision: [Nagihan İnan Gürcan].

\section{References}

1. Kumar V, Abbas AK, Fausto N et-al (2005) Robbins and Cotran pathologic basis of disease. W B Saunders Co. ISBN:0721601871

2. Fink KR, Fink JR (2013) Imaging of brain metastases. Surg Neurol Int. May 2;4(Suppl 4):S209-19

3. Boroughs LK, DeBerardinis RJ. Metabolic pathways promoting cancer cell survival and growth. Nat Cell Biol. 2015 Apr;17(4):351-9

4. Robertson-Tessi M, Gillies RJ, Gatenby RA, Anderson AR. Impact of metabolic heterogeneity on tumor growth, invasion, and treatment outcomes. Cancer Res. 2015 Apr 15;75(8):1567-79

5. Ganeshan B, Miles KA, Young RC, Chatwin CR (2007 Aug) Hepatic entropy and uniformity: additional parameters that can potentially increase the effectiveness of contrast enhancement during abdominal CT. Clin Radiol 62(8):761-768 
6. Ganeshan B, Abaleke S, Young RC, Chatwin CR, Miles KA. Texture analysis of non-small cell lung cancer on unenhanced computed tomography: initial evidence for a relationship with tumour glucose metabolism and stage. Cancer Imaging. 2010 Jul 6;10(1):137-43

7. Miles KA, Ganeshan B, Griffiths MR, Young RC, Chatwin CR (2009 Feb) Colorectal cancer: texture analysis of portal phase hepatic CT images as a potential marker of survival. Radiology 250(2):444452

8. Alic L, Niessen WJ, Veenland JF. Quantification of heterogeneity as a biomarker in tumor imaging: a systematic review. PLoS One. 2014 Oct 20;9(10):e110300

9. Stein D, Goldberg N, Domachevsky L, Bernstine H, Nidam M, Abadi-Korek I, Guindy M, Sosna J, Groshar D (2018) Quantitative biomarkers for liver metastases: comparison of MRI diffusionweighted imaging heterogeneity index and fluorine-18-fluoro-deoxyglucose standardised uptake value in hybrid PET/MR. Clin Radiol. Sep;73(9):832.e17-832.e22

10. Dong X, Wu P, Sun X, Li W, Wan H, Yu J, Xing L (2015 Jun) Intra-tumour 18F-FDG uptake heterogeneity decreases the reliability on target volume definition with positron emission tomography/computed tomography imaging. J Med Imaging Radiat Oncol 59(3):338-345

11. Yoon HJ, Kim Y, Kim BS (2015 Dec) Intratumoral metabolic heterogeneity predicts invasive components in breast ductal carcinoma in situ. Eur Radiol 25(12):3648-3658

12. Bundschuh RA, Dinges J, Neumann L et al (2014 Jun) Textural Parameters of Tumor Heterogeneity in 1â冈,F-FDG PET/CT for Therapy Response Assessment and Prognosis in Patients with Locally Advanced Rectal Cancer. J Nucl Med 55(6):891-897

13. Yankeelov TE, Peterson TE, Abramson RG, Izquierdo-Garcia D, Arlinghaus LR, Li X, Atuegwu NC, Catana C, Manning HC, Fayad ZA, Gore JC. Simultaneous PET-MRI in oncology: a solution looking for a problem? Magn Reson Imaging. 2012 Nov;30(9):1342-56

14. Punwani S, Taylor SA, Saad ZZ, Bainbridge A, Groves A, Daw S, Shankar A, Halligan S, Humphries PD (2013 Feb) Diffusion-weighted MRI of lymphoma: prognostic utility and implications for PET/MRI? Eur J Nucl Med Mol Imaging 40(3):373-385

15. Rohren EM, Provenzale JM, Barboriak DP, Coleman RE (2003 Jan) Screening for cerebral metastases with FDG PET in patients undergoing whole-body staging of non-central nervous system malignancy. Radiology 226(1):181-187

16. Wong CS, Gong N, Chu YC, Anthony MP, Chan Q, Lee HF, Chu KM, Khong PL (2012 Sep) Correlation of measurements from diffusion weighted MR imaging and FDG PET/CT in GIST patients: ADC versus SUV. Eur J Radiol 81(9):2122-2126

17. van Rijswijk CS, Geirnaerdt MJ, Hogendoorn PC, Peterse JL, van Coevorden F, Taminiau AH, Tollenaar RA, Kroon BB, Bloem JL (2003 Aug) Dynamic contrast-enhanced MR imaging in monitoring response to isolated limb perfusion in high-grade soft tissue sarcoma: initial results. Eur Radiol 13(8):18491858

18. Kong E, Chun KA, Cho IH. Quantitative assessment of simultaneous F-18 FDG PET/MRI in patients with various types of hepatic tumors: Correlation between glucose metabolism and apparent 
diffusion coefficient. PLoS One. 2017 Jul 3;12(7):e0180184

19. Just $\mathrm{N}(2014 \mathrm{Dec})$ Improving tumour heterogeneity MRI assessment with histograms. Br J Cancer 9(12):2205-2213 111(

20. Danoob D, Padhani AR, Grimm R. Quantitative WB-MRI with ADC Histogram Analysis for Response Assessment in Diffuse Bone Disease. Clinical Oncological Imaging. MAGNETOM Flash 2017 (69) $3 / 2017$

21. Rosenkrantz AB, Sigmund EE, Winnick A, Niver BE, Spieler B, Morgan GR, Hajdu CH (2012 Dec) Assessment of hepatocellular carcinoma using apparent diffusion coefficient and diffusion kurtosis indices: preliminary experience in fresh liver explants. Magn Reson Imaging 30(10):1534-1540

22. Rakheja R, Makis W, Skamene S, Nahal A, Brimo F, Azoulay L, Assayag J, Turcotte R, Hickeson M (2012 Jun) Correlating metabolic activity on 18F-FDG PET/CT with histopathologic characteristics of osseous and soft-tissue sarcomas: a retrospective review of 136 patients. AJR Am J Roentgenol 198(6):1409-1416

23. Nakajo M, Kajiya Y, Kaneko T, Kaneko Y, Takasaki T, Tani A, Ueno M, Koriyama C, Nakajo M (2010 Nov) FDG PET/CT and diffusion-weighted imaging for breast cancer: prognostic value of maximum standardized uptake values and apparent diffusion coefficient values of the primary lesion. Eur $\mathrm{J}$ Nucl Med Mol Imaging 37(11):2011-2020

24. Marusyk A, Polyak K (2010 Jan) Tumor heterogeneity: causes and consequences. Biochim Biophys Acta 1805(1):105-117

\section{Tables}

Table 1

Primary source and histopathology of metastases and distribution of the study population.

\begin{tabular}{|llll|}
\hline Primary Source & Histopathology & No. of patients & No. of metastases \\
\hline Breast & Invasive ductal & 4 & 11 \\
\hline \multirow{2}{*}{ Lung } & Invasive lobular & 4 & 9 \\
\hline \multirow{2}{*}{ Kidney } & Adenocarcinoma & 5 & 7 \\
Skin & Small cell carcinoma & 2 & 5 \\
\hline Total & Renal cell carcinoma & 2 & 2 \\
\hline
\end{tabular}


Table 2

A comparison between SUV and ADC data according to mean of three raters in normal appearing brain parenchyma and brain metastases.

\begin{tabular}{|llll|}
\hline Variable $(\mathbf{n}=\mathbf{4 0})$ & $\begin{array}{l}\text { Normal appearing brain } \\
\text { parenchyma }\end{array}$ & $\begin{array}{l}\text { Brain } \\
\text { metastases }^{\text {a }}\end{array}$ & $\begin{array}{c}\text { p-two } \\
\text { tailed }^{\mathbf{b}}\end{array}$ \\
\hline SUVmax & $3.33 \pm 1.13$ & $8.44 \pm 3.38$ & $<0.001$ \\
\hline $\begin{array}{l}\mathrm{ADCmean}\left(10^{-3}\right. \\
\left.\mathrm{mm}^{2} / \mathrm{s}\right)\end{array}$ & $0.82 \pm 0.07$ & $0.86 \pm 0.15$ & 0.253 \\
\hline $\mathrm{ADC}_{\mathrm{CV}}$ & $0.05 \pm 0.02$ & $0.17 \pm 0.06$ & $<0.001$ \\
\hline${ }^{*} \mathrm{SUV}$, standardized uptake value; ADC, apparent diffusion coefficient; CV, coefficient of variance \\
\hline a mean \pm Standard deviation & & \\
\hline b Wilcoxon's signed-rank test & & \\
\hline
\end{tabular}

Table 3

Receiver operating characteristics analysis

\begin{tabular}{|llllllll|}
\hline Variable & AUC & SE & $\begin{array}{l}\text { 95\% Confidence } \\
\text { interval }\end{array}$ & Sensitivity & Specificity & $\begin{array}{l}\text { Cut-off } \\
\text { value }\end{array}$ & p \\
\hline SUV & 0.961 & 0.024 & $0.913-1.000$ & $95 \%$ & $95 \%$ & $\geq 4.00$ & $<$ \\
\hline ADCmean & 0.574 & 0.068 & $0.441-0.707$ & $55 \%$ & $70 \%$ & 0.001 \\
\hline ADC $C$ CV & 0.998 & 0.003 & $0.992-1.000$ & $97.5 \%$ & $97.5 \%$ & $\geq 0.10$ & 0.256 \\
\hline $\begin{array}{l}\text { *AUC, area under the curve; SE, standard error; SUV, standardized uptake value; ADC, apparent } \\
\text { diffusion coefficient; CV, coefficient of variance }\end{array}$ & \\
\hline
\end{tabular}

\section{Figures}




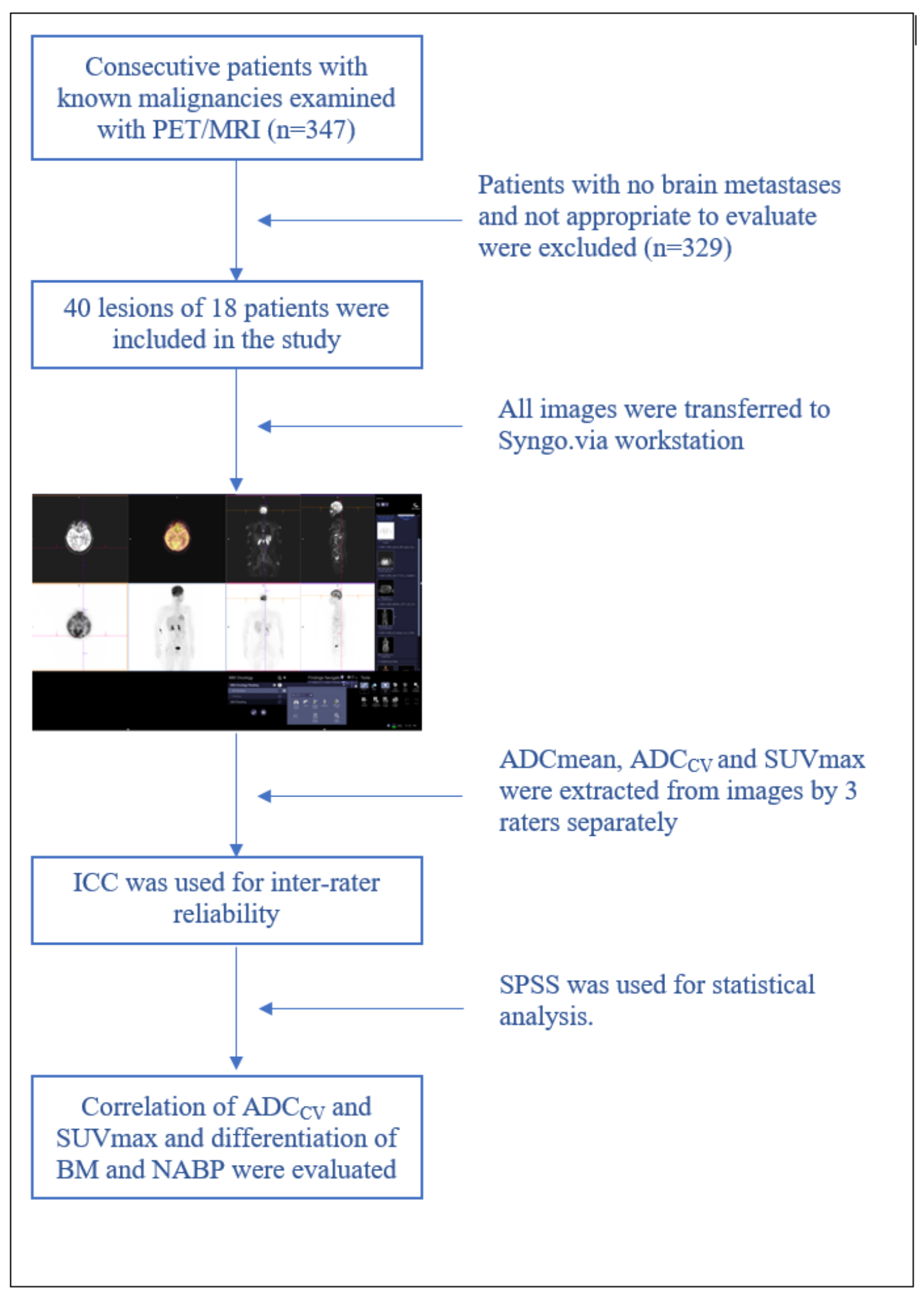

\section{Figure 1}

Design of the study * ADC, Apparent diffusion coefficient; CV, coefficient of variation; SUV, Standardized uptake value; ICC, Inter-class correlation coefficient; SPSS, Statistical package for the social sciences; BM, Brain metastases; NABP, Normal appearing brain parenchyma 

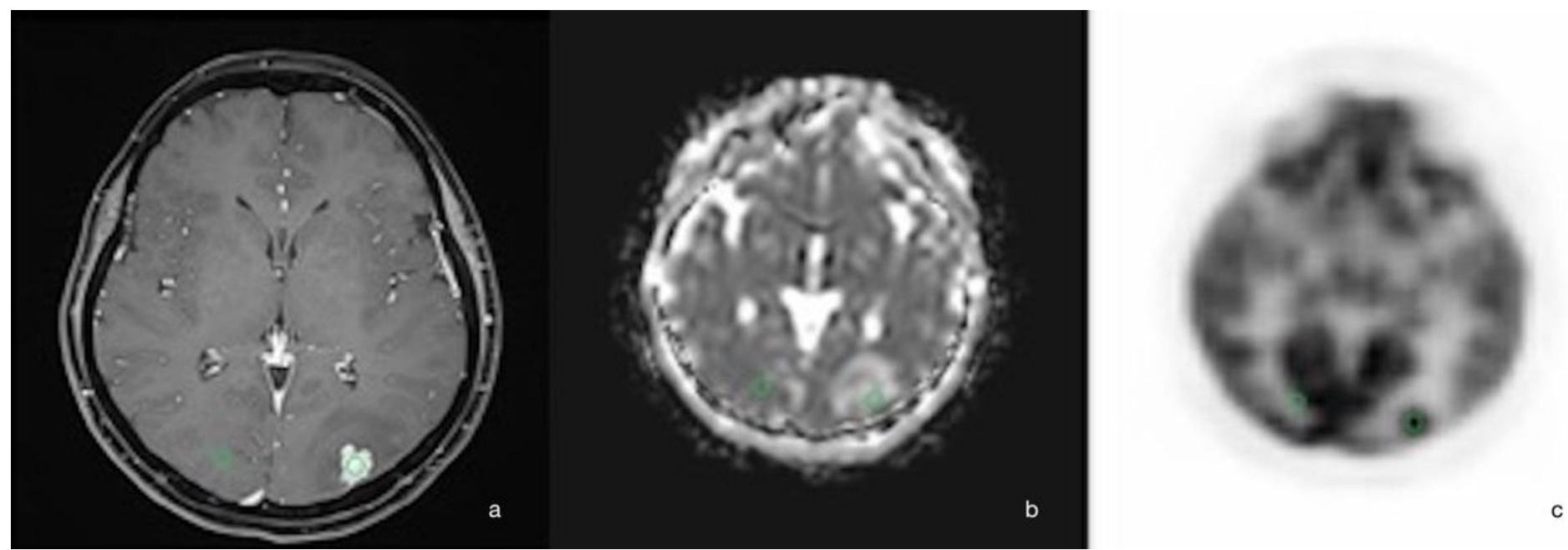

Figure 2

Replacing the VOI a) An axial contrast enhanced T1 weighted image was used for anatomical reference and two VOls with same size was drawn on brain metastasis and normal appearing brain parenchyma with giving care to tumor margins $b$ ) Both VOI was copied and placed with the same location on axial ADC images $\mathrm{c}$ ) The same process was repeated for the SUV-PET images on axial plan 